\title{
The Computer Olympiad 2020
}

\author{
Hiroyuki Iida ${ }^{\mathrm{a}, *, * *}$, Jonathan Schaeffer ${ }^{\mathrm{b}}$ and I-Chen $\mathrm{Wu}^{\mathrm{c}}$ \\ a Japan Advanced Institute of Science and Technology, Japan \\ ${ }^{\mathrm{b}}$ Department of Computing Science, University of Alberta, Canada T6G 2E8 \\ ${ }^{\mathrm{c}}$ Department of Computer Science, National Yang Ming Chiao Tung University, Hsinchu, Taiwan
}

\begin{abstract}
The $23^{\text {rd }}$ Computer Olympiad was held November/December 2020. As a consequence of the global pandemic, for the first time the competitions were fought online. This involved creating new rules that were, in large part, dependent on having the participants cooperate on organizing and conducting the events. The event was a success and will be repeated online in 2021.
\end{abstract}

\section{INTRODUCTION}

The pandemic has forced many events to move online, and the Computer Olympiad was no exception. Initially the Olympiad, Computers and Games conference, and World Computer Chess Championship were to be held in June 2020 in Santiago de Compostela, Spain, as part of the European Conference on Artificial Intelligence (ECAI). ECAI eventually was moved online and held at the end of August 2020. Unfortunately the timeline was too tight for the ICGA to move our events online in time to co-locate with ECAI. In the end, the Computer Olympiad was held online in November/December 2020, the Computers and Games conference was cancelled, and the World Computer Chess Championships cancelled. At the time of this writing, the 2021 Computer Olympiad will be held online in August, the Advances in Computer Games conference in November, and the World Computer Chess Championships are yet to be determined.

This report is a summary of the 2020 Computer Olympiad. Previously there had been 22 Olympiads going back to the first one in 1989. All had been face-to-face events. Moving online faced some challenges, but in the end, everything worked well and we had a successful event.

\section{PREPARATION}

The ICGA held off moving the Olympiad online, holding out hope for the usual in-person event (along with a conference and the chess championships). When reality set in, we quickly moved to the online format. A revised call for participation reflected the changes needed for the venue change (2020 Computer Olympiad, 2021).

Although we had concerns about our community's willingness to move from an in-person to an online format, they proved unfounded. Everyone recognized there was only one reasonable possibility - it was either an online Olympiad or no Olympiad.

\footnotetext{
*Research Center for Entertainment Science, Japan Advanced Institute of Science and Technology, 1-1 Asahidai, Nomi, Ishikawa 923-1292, Japan.

** Corresponding author. E-mail: iida@ jaist.ac.jp.
} 
The ideal scenario was that all competitions would move to the same online server. To facilitate that, the ICGA partnered with the LUDII project (Cameron Browne, project lead). "LUDII is a general game system designed to play, evaluate and design a wide range of games, including board games, card games, dice games, mathematical games, and so on" (Ludii, 2021). With some additional game support which the LUDII team enthusiastically carried out, the system offered us the opportunity to have a single uniform venue for the Olympiad. Unfortunately, this meant that Olympiad contestants would have to use the LUDII API (Application Programming Interface). This turned out to be a barrier for some (it meant additional programming) and alternatives were sought (the path of least effort).

The online event required creating an additional set of rules to govern the new format (2020 Computer Olympiad Rules, 2021). Essentially, we made each event to be self-organized by the participants. It could be held using LUDII, using an existing server (some games already had well-established servers), or some other communication method (e.g., playing using a video conferencing tool). As it turned out, most of the events were held using existing servers.

For each event, the participants were asked to agree on the following competition details (November 25-29):

- Format, with a round-robin being the standard.

- Number of games played between competitors.

- Time controls (most games had a standard setting).

- Playoffs. Some events agreed on the final round-robin standings serving for decisions on the medals. Other competitions had 1, 2 or 4-game matches to determine the final results.

Additional rules were introduced to accommodate the reality of online play, including lost Internet connections. Any disputes were to be handled by the three authors of this article.

Each event had 10 days to complete all the non-playoff games (November 30 to December 9), and an additional four days for playoffs (December 11-14). These dates slipped by a few days for some of the competitions.

\section{RESULTS}

Twenty-one competitions were held, with a total of 65 participants. In terms of the number of events, it was the third largest in Olympiad history, behind 24 in the 22 $2^{\text {nd }}$ Olympiad (Macau, 2019) and 22 in the $21^{\text {st }}$ Olympiad (Taipei, 2018). The number of participating programs was the $8^{\text {th }}$ in Olympiad history, behind 108 at the $15^{\text {th }}$ Olympiad (Kanazawa, Japan, 2010).

The event ran smoothly, with no disputes. It ran a few days longer than expected, the result of trying to accommodate people's schedules.

The ICGA congratulations all the Gold, Silver, and Bronze Medal winners, and thanks all the participants of the $23^{\text {rd }}$ Computer Olympiad. There were many interesting results, but two accomplishments stand out for special recognition:

- Quentin Cohen-Solal and Tristan Cazenave entered nine competitions, winning an impressive five Gold Medals and one Silver Medal.

- Ton Tillemams won his sixth Gold Medal in draughts $(10 \times 10$ checkers $)$.

Congratulations to Quentin, Tristan, and Ton! 
One lesson learned was to collect registration fees in advance. As of the day before the events began, one-third of the registrants had not paid. We started the competitions on time, rather than postponing them and inconveniencing everyone. It took another couple of months to collect the registration fees (one entrant never paid). This annoyance will be corrected for the next Olympiad.

The following gives the participants, competitive results, and medal winners for all the events. For a few of the events, there will be a separate in-depth report published in the ICGA Journal.

\section{Amazons}

8QP: Johan de Koning

SherlockGo: Yupeng Zhang, Liang Shuang, Liang Tailin, Wang Jilong, Li Xiaorui, Zhou Ke

Thésée: Quentin Cohen-Solal, Tristan Cazenave

\begin{tabular}{|l|c|c|c|c|}
\hline Program & $8 Q P$ & SherlockGo & Thésée & Total \\
\hline $8 Q P$ & $\mathbf{X}$ & 2 & 0 & $\mathbf{2}$ \\
\hline SherlockGo & 0 & $\mathbf{X}$ & 0 & $\mathbf{0}$ \\
\hline Thésée & 2 & 2 & $\mathbf{X}$ & $\mathbf{4}$ \\
\hline
\end{tabular}

Congratulations to Quentin Cohen-Solal and Tristan Cazenave, authors of Thésée, for winning the Gold Medal in the 2020 Computer Olympiad in Amazons.

Congratulations to Johan de Koning, author of $8 Q P$, for winning the Silver Medal in the 2020 Computer Olympiad in Amazons.

\section{Breakthrough}

DaSoJai: Yen-Chi Chen, Wei-Lin Wu, Shun-Shii Lin

NDHU Polygames: Akeem Peters, Facebook, NDHU

R2D2: Quentin Cohen-Solal, Tristan Cazenave

\begin{tabular}{|l|c|c|c|c|}
\hline Program & DaSoJai & NDHU Polygames & R2D2 & Total \\
\hline DaSoJai & $\mathbf{X}$ & 0 & 0 & $\mathbf{0}$ \\
\hline NDHU Polygames & 2 & $\mathbf{X}$ & 0 & $\mathbf{2}$ \\
\hline$R 2 D 2$ & 2 & 2 & $\mathbf{X}$ & $\mathbf{4}$ \\
\hline
\end{tabular}


Congratulations to Quentin Cohen-Solal and Tristan Cazenave, authors of $R 2 D 2$, for winning the Gold Medal in the 2020 Computer Olympiad in Breakthrough.

Congratulations to Akeem Peters (Facebook, NDHU), author of NDHU Polygames, for winning the Silver Medal in the 2020 Computer Olympiad in Breakthrough.

\section{Bridge (HoneyMoon)}

BridgeYeh: Jyun-Tin Yeh, Shun-Shii Lin

CYC_HB: YiChuan Chen

GodJimmy: Chen-En Yang, Yen-Chi Chen, Shun-Shii Lin

\begin{tabular}{|l|c|c|c|c|}
\hline Program & BridgeYeh & CYC_HB & GodJimmy & Total \\
\hline BridgeYeh & $\mathbf{X}$ & 43 & 26 & $\mathbf{6 9}$ \\
\hline CYC_HB & 57 & $\mathbf{X}$ & 28 & $\mathbf{8 5}$ \\
\hline GodJimmy & 74 & 72 & $\mathbf{X}$ & $\mathbf{1 4 6}$ \\
\hline
\end{tabular}

Congratulations to Chen-En Yang, Yen-Chi Chen, Shun-Shii Lin, authors of GodJimmy, for winning the Gold Medal in the 2020 Computer Olympiad in Honeymoon Bridge.

Congratulations to YiChuan Chen, author of $C Y C_{-} H B$, for winning the Silver Medal in the 2020 Computer Olympiad in Honeymoon Bridge.

\section{Chess (Chinese Dark)}

DarkCheese: Ping-Chun Chen

DarkKnight: Chu-Hsuan Hsueh, Wen-Jie Tseng, I-Chen Wu

PupilDarkChess: Cheng Yueh, Hung-Jui Chang

Unknow: Yu-Yan Wang

Yanyu2.0: Yu-Yan Wang 


\begin{tabular}{|l|c|c|c|c|c|c|}
\hline Program & DarkCheese & DarkKnight & PupilDark Chess & Unknow & Yanyu2.0 & Total \\
\hline DarkCheese & $\mathbf{X}$ & 0.0 & 0.5 & 2.0 & 2.0 & $\mathbf{4 . 5}$ \\
\hline DarkKnight & 2.0 & $\mathbf{X}$ & 0.0 & 2.0 & 1.5 & $\mathbf{5 . 5}$ \\
\hline PupilDarkChess & 1.5 & 2.0 & $\mathbf{X}$ & 2.0 & 1.5 & $\mathbf{7 . 0}$ \\
\hline Unknow & 0.0 & 0.0 & 0.0 & $\mathbf{X}$ & 0.5 & $\mathbf{0 . 5}$ \\
\hline Yanyu2.0 & $0 . .0$ & 0.5 & 0.5 & 1.5 & $\mathbf{X}$ & $\mathbf{2 . 5}$ \\
\hline
\end{tabular}

Congratulations to Cheng Yueh and Hung-Jui Chang, authors of PupilDarkChess, for winning the Gold Medal in the 2020 Computer Olympiad in Chinese Dark Chess.

Congratulations to Chu-Hsuan Hsueh, Wen-Jie Tseng, and I-Chen Wu, authors of DarkKnight, for winning the Silver Medal in the 2020 Computer Olympiad in Chinese Dark Chess.

Congratulations to Ping-Chun Chen, author of DarkCheese, for winning the Bronze Medal in the 2020 Computer Olympiad in Chinese Dark Chess.

\section{Chinese Checkers}

Hexagram: Zaheen Ahmad, Nathan Sturtevant

Jump: Yen-Chi, Jiunn-Haur Chern, Shun-Shii Lin

Negentropy: Yen-Chi, Lu-Nung Chen, Shun-Shii Lin

\begin{tabular}{|l|c|c|c|c|}
\hline Program & Hexagram & Jump & Negentropy & Total \\
\hline Hexagram & $\mathbf{X}$ & 0 & 2 & $\mathbf{2}$ \\
\hline Jump & 2 & $\mathbf{X}$ & 2 & $\mathbf{4}$ \\
\hline Negentropy & 0 & 0 & $\mathbf{X}$ & $\mathbf{0}$ \\
\hline
\end{tabular}

Congratulations to Yen-Chi, Jiunn-Haur Chern, and Shun-Shii Lin, authors of Jump, for winning the Gold Medal in the 2020 Computer Olympiad in Chinese Checkers.

Congratulations to Zaheen Ahmad and Nathan Sturtevant, authors of Hexagram, for winning the Silver Medal in the 2020 Computer Olympiad in Chinese Checkers. 


\section{Clobber}

Calpurnia: Christian Jans

Deep Nikita: Andrew Lin

Hercule: Quentin Cohen-Solal, Tristan Cazenave

Klopper: Johannes Schwagereit

Pan: Johan de Koning

\begin{tabular}{|l|c|c|c|c|c|c|}
\hline Program & Calpurnia & Deep Nikita & Hercule & Klopper & Pan & Total \\
\hline Calpurnia & $\mathbf{X}$ & $0-2$ & $0-2$ & $1-1$ & $0-2$ & $\mathbf{1}$ \\
\hline Deep Nikita & $2-0$ & $\mathbf{X}$ & $0-0$ & $0-0$ & $1-1$ & $\mathbf{3}$ \\
\hline Hercule & $2-0$ & $0-0$ & $\mathbf{X}$ & $2-0$ & $1-1$ & $\mathbf{5}$ \\
\hline Klopper & $1-1$ & $0-0$ & $0-2$ & $\mathbf{X}$ & $0-2$ & $\mathbf{1}$ \\
\hline Pan & $2-0$ & $1-1$ & $1-1$ & $2-0$ & $\mathbf{X}$ & $\mathbf{6}$ \\
\hline
\end{tabular}

Deep Nikita was unable to complete the tournament.

Playoff \#1: Hercule 2 - Klopper 0. Hercule moves on to the Gold Medal game. Klopper wins the Bronze Medal.

Playoff \#2: Pan 1 - Hercule 2. Hercule wins the Gold Medal. Pan wins the Silver Medal.

Congratulations to Quentin Cohen-Solal and Tristan Cazenave, authors of Hercule, for winning the Gold Medal in the 2020 Computer Olympiad in Clobber.

Congratulations to Johan de Koning, author of Pan, for winning the Silver Medal in the 2020 Computer Olympiad in Clobber.

Congratulations to Johannes Schwagereit, author of Klopper, for winning the Bronze Medal in the 2020 Computer Olympiad in Clobber.

\section{Connect6}

CZF_Connect6: Shao-Xiong Zheng, Tzu-Yee Yang, I-Chen Wu

Explorer: Xiaorui Li, Yunpeng Zhang, Yaguang Li, Yihao Wu, Miao Su, Ke Zhou

Kavalan: Jung-Kuei Yang

NDHU Polygames: Sasa Tarzn, Facebook, NDHU 


\begin{tabular}{|l|c|c|c|c|c|}
\hline Program & CZF_Connect6 & Explorer & Kavalan & NDHU Polygames & Total \\
\hline CZF_Connect6 & $\mathbf{X}$ & 2 & 2 & 1 & $5(2)$ \\
\hline Explorer & 0 & $\mathbf{X}$ & 2 & 0 & 2 \\
\hline Kavalan & 0 & 0 & $\mathbf{X}$ & 0 & 0 \\
\hline NDHU Polygames & 1 & 2 & 2 & $\mathbf{X}$ & $5(0)$ \\
\hline
\end{tabular}

A two-game match was used to decide on the Gold Medal.

Congratulations to Shao-Xiong Zheng, Tzu-Yee Yang, and I-Chen Wu, authors of CZF_Connect6, for winning the Gold Medal in the 2020 Computer Olympiad in Connect6.

Congratulations to Sasa Tarzn (Facebook, NDHU), author of NDHU Polygames, for winning the Silver Medal in the 2020 Computer Olympiad in Connect6.

Congratulations to Xiaorui Li, Yunpeng Zhang, Yaguang Li, Yihao Wu, Miao Su, and Ke Zhou, authors of Explorer, for winning the Bronze Medal in the 2020 Computer Olympiad in Connect6.

\section{Dots and Boxes}

Deep Nikia: Andrew Lin

Control Freak: William Fraser

\begin{tabular}{|l|c|c|}
\hline Program & Deep Nikita & Control Freak \\
\hline Deep Nikita & $\mathbf{X}$ & $1-1$ \\
\hline Control Freak & $0-0$ & $\mathbf{X}$ \\
\hline
\end{tabular}

Congratulations to Andrew Lin, author of Deep Nikita, for winning the Gold Medal in the 2020 Computer Olympiad in Dots and Boxes.

\section{Draughts (International)}

TDKing: Ton Tillemans

Tornado: Frank Mesander 


\begin{tabular}{|l|c|c|c|}
\hline Program & TDking & Tornado & Points \\
\hline TDking & $\mathbf{X}$ & $2-1-1-1-1-1$ & $\mathbf{7}$ \\
\hline Tornado & $0-1-1-1-1-1$ & $\mathbf{X}$ & $\mathbf{5}$ \\
\hline
\end{tabular}

Congratulations to Ton Tillemans, author of TDking, for winning the Gold Medal in the 2020 Computer Olympiad in Draughts (International).

\section{EinStein Würfelt Nicht}

NDHU Polygames: Yi-Chuan Chen, Facebook, NDHU

Reinstein: Zih-Yang Hsu, Chih-Lin Chen, Chia-Jung Hsu, Chun-Wei Chang

VSWTN: Yunpeng Zhang, Wei Li, Yuxuan Zhang, Ke Zhou

\begin{tabular}{|l|c|c|c|c|}
\hline Program & NDHU Polygames & Reinstein & VSWTN & Points \\
\hline NDHU Polygames & $\mathbf{X}$ & 7 & 6 & $\mathbf{1 3}$ \\
\hline Reinstein & 5 & $\mathbf{X}$ & 1 & $\mathbf{6}$ \\
\hline VSWTN & 6 & 11 & $\mathbf{X}$ & $\mathbf{1 7}$ \\
\hline
\end{tabular}

Congratulations to Yunpeng Zhang, Wei Li, Yuxuan Zhang, and Ke Zhou, authors of VSWTN, for winning the Gold Medal in the 2020 Computer Olympiad in EinStein Würfelt Nicht.

Congratulations to Yi-Chuan Chen (Facebook, NDHU), author of NDHU Polygames, for winning the Silver Medal in the 2020 Computer Olympiad in EinStein Würfelt Nicht.

Go $(9 \times 9)$

CGI Go Intelligence: Ti-Rong Wu, Guan-Wen Chen, Hsiao-Chung Hsieh, Chung-Chin Shih, MengYu Tsai, Yu-Shan Hsu, Chun-Jung Chen, I-Chen Wu

EzGo: Yi-Ting Chiang, Hung-Cheng Lin 


\begin{tabular}{|l|c|c|}
\hline Program & CGI Go Intelligence & EzGo \\
\hline CGI Go Intelligence & $\mathbf{X}$ & 4 \\
\hline$E z G o$ & 0 & $\mathbf{X}$ \\
\hline
\end{tabular}

Congratulations to Ti-Rong Wu, Guan-Wen Chen, Hsiao-Chung Hsieh, Chung-Chin Shih, Meng-Yu Tsai, Yu-Shan Hsu, Chun-Jung Chen, and I-Chen Wu, authors of CGI Go Intelligence, for winning the Gold Medal in the 2020 Computer Olympiad in Go $(9 \times 9)$.

\section{Gomoku (Outer-Open)}

CZF_Outer_Open_Gomoku: Wei-Chen Liao, Po-Wei Huang, Shao-Xiong Zheng, Yuan-Hao Chen, I-Chen $\mathrm{Wu}$

Fiver: Zhang Yiyang, Zhang Yunpeng, Xu Yanrui, Hao Ru, Liang Tailin, Zhou Ke

Nonoku: Jiunn-Haur Chern, Shun-Shii Lin

OOG: Chih-Hung Chen, Yen-Chi Chen, Shun-Shii Lin

\begin{tabular}{|l|c|c|c|c|c|}
\hline Program & CZF_Outer_Open_Gomoku & Fiver & Nonoku & OOG & Total \\
\hline CZF_Outer_Open_Gomoku & $\mathbf{X}$ & 2 & 2 & 2 & $\mathbf{6}$ \\
\hline Fiver & 0 & $\mathbf{X}$ & 2 & 1 & $\mathbf{3}$ \\
\hline Nonoku & 0 & 0 & $\mathbf{X}$ & 2 & $\mathbf{2}$ \\
\hline OOG & 0 & 1 & 0 & $\mathbf{X}$ & $\mathbf{1}$ \\
\hline
\end{tabular}

Congratulations to Wei-Chen Liao, Po-Wei Huang, Shao-Xiong Zheng, Yuan-Hao Chen, and I-Chen Wu, authors of CZF_Outer_Open_Gomoku, for winning the Gold Medal in the 2020 Computer Olympiad in Gomoku (Outer-Open).

Congratulations to Zhang Yiyang, Zhang Yunpeng, Xu Yanrui, Hao Ru, Liang Tailin, and Zhou Ke, authors of Fiver, for winning the Silver Medal in the 2020 Computer Olympiad in Gomoku (OuterOpen).

Congratulations to Jiunn-Haur Chern and Shun-Shii Lin, authors of Nonoku, for winning the Bronze Medal in the 2020 Computer Olympiad in Gomoku (Outer-Open).

Havannah $(8 \times 8)$

Doombot-8: Quentin Cohen-Solal, Tristan Cazenave 
NDHU Polygames: Tyler Eck, Facebook, NDHU

\begin{tabular}{|l|c|c|}
\hline Program & Doombot-8 & NDHU Polygames \\
\hline Doombot-8 & $\mathbf{X}$ & $0-0$ \\
\hline NDHU Polygames & $1-1$ & $\mathbf{X}$ \\
\hline
\end{tabular}

Congratulations to Tyler Eck (Facebook, NDHU), authors of NDHU Polygames, for winning the Gold Medal in the 2020 Computer Olympiad in Havannah $(8 \times 8)$.

Hex $(13 \times 13)$

NDHU Polygames: Sasa Tarzn, Facebook, NDHU

Ultron13: Quentin Cohen-Solal, Tristan Cazenave

\begin{tabular}{|l|c|c|}
\hline Program & NDHU Polygames & Ultron13 \\
\hline NDHU Polygames & $\mathbf{X}$ & $1-1$ \\
\hline Ultron13 & $0-0$ & $\mathbf{X}$ \\
\hline
\end{tabular}

Congratulations to Sasa Tarzn (Facebook, NDHU), author of NDHU Polygames, for winning the Gold Medal in the 2020 Computer Olympiad in Hex $(13 \times 13)$.

Hex $(19 \times 19)$

NDHU Polygames: Sasa Tarzn, Facebook, NDH

Ultron19: Quentin Cohen-Solal, Tristan Cazenave

\begin{tabular}{|l|c|c|}
\hline Program & NDHU Polygames & Ultron13 \\
\hline NDHU Polygames & $\mathbf{X}$ & 2 \\
\hline Ultron13 & 0 & $\mathbf{X}$ \\
\hline
\end{tabular}

Congratulations to Sasa Tarzn (Facebook, NDHU), author of NDHU Polygames, for winning the Gold Medal in the 2020 Computer Olympiad in Hex $(19 \times 19)$.

\section{Mahjong}

SimCat: Shih-Chieh Tang, Fong-Shen Liao, I-Chen Wu 
VeryLongCat: Shih-Chieh Tang, Li-Kai Chuang, I-Chen Wu

Zei: Yu-Chen Lin, Shun-Shii Lin

Zio: Zong-Han Lin, Yu-Chen Lin, Shun-Shii Lin

\begin{tabular}{|l|c|}
\hline Program & Score \\
\hline SimCat & $+\mathbf{1 0 8 , 5 0 0}$ \\
\hline VeryLongCat & $\mathbf{- 7 6 , 5 0 0}$ \\
\hline Zei & $\mathbf{- 6 1 , 0 0 0}$ \\
\hline Zio & $+\mathbf{2 9 , 0 0 0}$ \\
\hline
\end{tabular}

Congratulations to Shih-Chieh Tang, Fong-Shen Liao, and I-Chen Wu, authors of SimCat, for winning the Gold Medal in the 2020 Computer Olympiad in Mahjong.

Congratulations to Zong-Han Lin, Yu-Chen Lin, and Shun-Shii Lin, authors of Zio, for winning the Silver Medal in the 2020 Computer Olympiad in Mahjong.

Congratulations to Yu-Chen Lin and Shun-Shii Lin, authors of Zei, for winning the Bronze Medal in the 2020 Computer Olympiad in Mahjong.

\section{Nonogram}

The competition is to be the fastest solver of 1,000 problems.

Defense: Jia-Jun Yeh, Kuo-Chan Huang, Wei-Chiao Huang, Yan-Rong Guo, De-Rong Sung, TzungShuan Tsai

Requim: Yen-Chi Chen, Shun-Shii Lin

The Heirs: Jia-Jun Yeh, Kuo-Chan Huang,Wei-Chiao Huang, Yan-Rong Guo, Shao-Xiong Zheng, Tzung-Shuan Tsai

\begin{tabular}{|l|c|c|}
\hline Program & Problems Solved & Time (seconds) \\
\hline The Heirs & $\mathbf{1 , 0 0 0}$ & $\mathbf{3 7 0 . 5 6 8}$ \\
\hline Defense & $\mathbf{1 , 0 0 0}$ & $\mathbf{3 8 9 . 8 1 7}$ \\
\hline Requiem & $\mathbf{1 , 0 0 0}$ & $\mathbf{4 3 2 . 8 9 5}$ \\
\hline
\end{tabular}


Congratulations to Jia-Jun Yeh, Kuo-Chan Huang, Wei-Chiao Huang, Yan-Rong Guo, Shao-Xiong Zheng, and Tzung-Shuan Tsai, authors of The Heirs, for winning the Gold Medal in the 2020 Computer Olympiad in Nonogram.

Congratulations to Jia-Jun Yeh, Kuo-Chan Huang, Wei-Chiao Huang, Yan-Rong Guo, De-Rong Sung, and Tzung-Shuan Tsai, authors of Defense, for winning the Silver Medal in the 2020 Computer Olympiad in Nonogram.

\section{Othello $(8 \times 8)$}

Maverick: Yen-Chi Chen, Shun-Shii Lin

NDHU Polygames: Astrid Adhipurusa, Facebook, NDHU

Réplicateur \#8: Quentin Cohen-Solal, Tristan Cazenave

\begin{tabular}{|l|c|c|c|c|}
\hline Program & Maverick & NDHU Polygames & Réplicateur \#8 & Total \\
\hline Maverick & $\mathbf{X}$ & $1-1$ & $1-1$ & $\mathbf{4}$ \\
\hline NDHU Polygames & $0-0$ & $\mathbf{X}$ & $0-0$ & $\mathbf{0}$ \\
\hline Réplicateur \#8 & $0-0$ & $1-1$ & $\mathbf{X}$ & $\mathbf{2}$ \\
\hline
\end{tabular}

Congratulations to Yen-Chi Chen and Shun-Shii Lin, authors of Maverick, for winning the Gold Medal in the 2020 Computer Olympiad in Othello $(8 \times 8)$.

Congratulations to Quentin Cohen-Solal and Tristan Cazenave, authors of Réplicateur \#8, for winning the Silver Medal in the 2020 Computer Olympiad in Othello $(8 \times 8)$.

Othello $(10 \times 10)$

NDHU Polygames: Astrid Adhipurusa, Facebook, NDHU

Persona: Yen-Chi Chen, Surag Nair, Nai-Yuan Chang, Shun-Shii Lin

Réplicateur \#10: Quentin Cohen-Solal, Tristan Cazenave

\begin{tabular}{|l|c|c|c|c|}
\hline Program & NDHU Polygames & Persona & Réplicateur \#10 & Total \\
\hline NDHU Polygames & $\mathbf{X}$ & 1 & 0 & $\mathbf{1}(\mathbf{1})$ \\
\hline Persona & 1 & $\mathbf{X}$ & 0 & $\mathbf{1}(\mathbf{3})$ \\
\hline Réplicateur \#10 & 2 & 2 & $\mathbf{X}$ & $\mathbf{4}$ \\
\hline
\end{tabular}


A four-game match was used to decide on the Silver Medal.

Congratulations to Quentin Cohen-Solal and Tristan Cazenave, authors of Réplicateur \#10, for winning the Gold Medal in the 2020 Computer Olympiad in Othello $(10 \times 10)$.

Congratulations to Yen-Chi Chen, Surag Nair, Nai-Yuan Chang, and Shun-Shii Lin, authors of Persona, for winning the Silver Medal in the 2020 Computer Olympiad in Othello $(10 \times 10)$.

\section{Shogi (Mini)}

NDHU Polygames: Tyler Eck, Facebook, NDHU

Nyanpass: Hsuan-Yu Wang

ShioRamen: Masahiro Shioda, Takeshi Ito

\begin{tabular}{|l|c|c|c|c|}
\hline Program & NDHU Polygames & Nyanpass & ShioRamen & Total \\
\hline NDHU Polygames & $\mathbf{X}$ & 1 & 0 & $\mathbf{1}(\mathbf{2})$ \\
\hline Nyanpass & 1 & $\mathbf{X}$ & 0 & $\mathbf{1}(\mathbf{0})$ \\
\hline ShioRamen & 2 & 2 & $\mathbf{X}$ & $\mathbf{4}$ \\
\hline
\end{tabular}

A two-game match was used to decide on the Silver Medal.

Congratulations to Masahiro Shioda and Takeshi Ito, authors of ShioRamen, for winning the Gold Medal in the 2020 Computer Olympiad in Mini Shogi

Congratulations to Tyler Eck (Facebook, NDHU), author of NDHU Polygames, for winning the Silver Medal in the 2020 Computer Olympiad in Mini Shogi.

\section{Surakarta}

Athénan: Quentin Cohen-Solal, Tristan Cazenave

CZF_Surakarta: Liang-Fu Liu

FuChou: Jia-Fong Yeh, Yen-Chi Chen, Shun-Shii Lin

VSSurakarta: Zhang Yunpeng, Li Wei, Zhang Yuxuan, Zhang Pei, Zhou Ke 


\begin{tabular}{|l|c|c|c|c|c|}
\hline Program & Athénan & CZF_Surakarta & FuChou & VSSurakarta & Total \\
\hline Athénan & $\mathbf{X}$ & 1 & 1.5 & 2 & $\mathbf{4 . 5}$ \\
\hline CZF_Surakarta & 1 & $\mathbf{X}$ & 1 & 2 & $\mathbf{4}$ \\
\hline FuChou & 0.5 & 1 & $\mathbf{X}$ & 2 & $\mathbf{3 . 5}$ \\
\hline VSSurakarta & 0 & 0 & 0 & $\mathbf{X}$ & $\mathbf{0}$ \\
\hline
\end{tabular}

Congratulations to Quentin Cohen-Solal and Tristan Cazenave, authors of Athénan, for winning the Gold Medal in the 2020 Computer Olympiad in Surakarta.

Congratulations to Liang-Fu Liu, author of CZF_Surakarta, for winning the Silver Medal in the 2020 Computer Olympiad in Surakarta.

Congratulations to Jia-Fong Yeh, Yen-Chi Chen, and Shun-Shii Lin, authors of FuChou, for winning the Bronze Medal in the 2020 Computer Olympiad in Surakarta.

\section{CONCLUSIONS}

The 2020 Computer Olympiad was a success. This was only possible because of the cooperation of all of the participants. We are deeply appreciative of their support.

The 2021 Computer Olympiad, number 24 in the series, will begin at the end of November (2021 Computer Olympiad, 2021). We hope that you will consider participating in it!

For 2022, it appears likely that an in-person meeting will be possible. Whether we will go back to the event's 1989-2019 face-to-face format, continue with the 2020-2021 online version, or adopt a hybrid approach remains to be seen. Your thoughts on the matter are most welcome!

\section{REFERENCES}

2020 Computer Olympiad, https://icga.org/?page_id=3131.

2020 Computer Olympiad Rules. https://icga.org/?page_id=3146.

2021 Computer Olympiad, https://icga.org/?page_id=3216.

Ludii, http://ludii.games. 